\title{
COMMENTARY
}

\section{Biomarkers for late-onset neonatal sepsis}

\author{
Richard A Polin* and Tara M Randis
}

\begin{abstract}
The diagnosis of healthcare-associated infections is problematic because of the overlap between clinical signs associated with 'normal' physiological disturbances and those of bacteremia or fungemia. Earlier diagnosis of sepsis in critically ill infants would enable timely administration of antibiotics and discontinuation of treatment in infants with a low probability of sepsis. A recent study by $\mathrm{Ng}$ et al. identified two novel biomarkers for late-onset neonatal sepsis: the des-arginine variant of serum amyloid A and apolipoprotein C-II. These markers may be of value in the identification of neonates with bacteremia or fungemia.
\end{abstract}

\section{Background}

Late-onset sepsis in newborn infants is defined as sepsis occurring after the first 72 hours of life and is a major cause of infant mortality [1]. Furthermore, these infections increase the length of hospital stay, add millions of dollars in excess healthcare costs annually and are associated with poorer neurodevelopmental outcomes. Infants of any gestational age are susceptible to late-onset sepsis. However, very low birth weight infants (those weighing less than $1,500 \mathrm{~g}$ ) are particularly vulnerable because of the need for invasive monitoring, impaired host defense mechanisms, limited amounts of normal endogenous flora, reduced barrier function of neonatal skin and frequent exposure to broad-spectrum antibiotics. Most late-onset infections in newborn infants are classified as healthcare-associated infections because they occur while these infants are receiving neonatal intensive care. The two most common presentations are catheter-associated bloodstream infections and ventilatorassociated pneumonia. The diagnosis of healthcareassociated infections is problematic because of the overlap between clinical signs associated with 'normal'

*Correspondence: rap32@columbia.edu

Columbia University College of Physicians and Surgeons, Division of Neonatology, NY Presbyterian Morgan Stanley Children's Hospital, 3959 Broadway, CHC 115, New York, NY 10032, USA physiological disturbances and those of bacteremia or fungemia. Earlier diagnosis of sepsis in critically ill infants would enable timely administration of antibiotics and discontinuation of treatment in infants with a low probability of sepsis.

\section{Biomarker discovery}

The use of plasma proteomics in biomarker discovery has been met with limited success. A recent review of the subject by Lottspeich et al. [2] suggests several reasons for this, including individual heterogeneity due to genetic and environmental factors, technical complexity of quantitative plasma protein analysis and lack of reproducibility in blood sampling specimen processing. Despite these limitations, Ng et al. [3] present an intriguing study using proteomic analysis to identify novel host-response biomarkers for early diagnosis of late-onset sepsis and necrotizing enterocolitis in preterm infants. The authors used a rigorous scientific study design, comprising both a case-control discovery and initial validation phase and a secondary prospective cohort validation phase, in order to minimize the inherent systemic biases that often plague biomarker discovery trials. Furthermore, the discovery phase of this study [3] included a longitudinal analysis of candidate biomarkers to demonstrate reversal of expression patterns over time, thereby verifying the pathophysiological relevance of these proteins. Initial validation studies identified serum amyloid A (SAA) and apolipoprotein $\mathrm{C}$-II as the most promising candidate markers. Both plasma proteins may be mechanistically linked to the disease process (lipoprotein modulation of host immune response) so are biologically plausible as candidate biomarkers. Because plasma concentrations of these biomarker proteins may be determined using highthroughput immunoassay techniques, incorporation of diagnostic tests for them into clinical practice is economically and technically feasible. A diagnostic equation was created (the ApoSAA score) and validated both statistically (Hosmer-Lemeshow goodness of fit test) and clinically in a prospective cohort study. The authors [3] used this score to stratify very low birth weight infants by risk for sepsis and/or necrotizing enterocolitis, and they developed clinical guidelines for the initiation and timely discontinuation of antibiotics for each risk category. 
There are some potential limitations of this study that warrant further investigation. Experimental data suggest that SAA levels may be affected by nutritional status and hepatic function $[4,5]$. In addition, infusion of lipid emulsions in parenteral nutrition has been associated with modification of apolipoprotein C-II [6]. These potential confounding variables are particularly problematic in a patient population in which parenteral nutrition, cholestatic liver disease and postnatal growth failure are very common.

\section{Apolipoprotein C-II and serum amyloid A}

It is interesting that $\mathrm{Ng}$ et al. [3] identified two markers of late-onset sepsis involved in lipid metabolism. Serum amyloid A proteins are a family of apolipoproteins associated with high-density lipoprotein in plasma; apolipoprotein C-II is responsible for activation of lipoprotein lipase. High-density lipoproteins have been shown to bind bacterial cell wall components, including lipoteichoic acid and endotoxin $[7,8]$. That suggests a role for lipoproteins in modulating the immune response. It is well known that circulating levels of free fatty acids, triglycerides and cholesterol are increased during sepsis. This response is beneficial to the infected host because lipids provide an alternative energy source for injured tissues. The synthesis of apolipoproteins is increased in the liver with sepsis; however, gene expression of lipoprotein lipase is downregulated by tumor necrosis factor- $\alpha$ (TNF- $\alpha)$ and other cytokines.

Serum amyloid A is a generic term for a family of proteins that is used as a marker of acute and chronic inflammation $[9,10]$. The dominant isotype (SAA1) consists of at least five allelic variants. SAA is believed to be a more sensitive indicator of inflammation than the widely used C-reactive protein. Expression of SAA is principally regulated at the transcriptional level by cytokines (TNF- $\alpha$ and interleukins 1 and 6) or glucocorticoids. SAA levels peak on the third day after a sepsis episode and return to baseline approximately 4 days later. Several other investigators have studied the utility of SAA in the diagnosis of late-onset sepsis [11]. SAA is believed to rise more rapidly than C-reactive protein and may have a better prognostic value during the first 24 hours after sepsis onset. Furthermore, SAA has a high negative predictive value and, therefore, might be useful in identifying infants with a low probability of sepsis. The des-arginine variant of SAA, used by $\mathrm{Ng}$ et $a l$., has been investigated in other infectious diseases [12], but not previously in neonatal sepsis. Apolipoprotein C-I has been shown to correlate with mortality in adults with sepsis, but not apolipoprotein C-II. The study by Ng et al. [3] is the first to investigate apolipoprotein $\mathrm{C}$-II in neonatal sepsis and necrotizing enterocolitis.

\section{Conclusions}

Blood culture remains the diagnostic gold standard for late-onset sepsis. However, clinicians frequently rely on screening tests, such as the white blood cell count, immature to total neutrophil ratio and C-reactive protein, to guide decisions regarding initiation or discontinuation of antimicrobial therapy. Because of the morbidity and potential mortality associated with late-onset sepsis, screening tests with high sensitivity and negative predictive value are most valuable because all infected infants must be identified, and infants without sepsis should be spared antimicrobial therapy. The extraordinarily high sensitivity and negative predictive value of the ApoSAA score proposed by $\mathrm{Ng}$ et al. [3] make this screening strategy an appealing option. However, the generalizability of this tool to wider patient populations remains unknown. External validation studies assessing the performance of these biomarkers in other institutions and in infants of varying gestational ages are necessary before widespread adoption into clinical practice is considered.

\section{Abbreviations}

ApoSAA, apolipoprotein serum amyloid A; SAA, serum amyloid A; TNF, tumor necrosis factor.

\section{Competing interests}

The authors have no competing interests to declare.

\section{Authors' contributions}

Both authors contributed equally to the writing of the manuscript.

\section{Published: 6 September 2010}

\section{References}

1. Stoll BJ, Hansen N, Fanaroff AA, Wright LL, Carlo WA, Ehrenkranz RA, Lemons JA, Donovan EF, Stark AR, Tyson JE, Oh W, Bauer CR, Korones SB, Shankaran S, Laptook AR, Stevenson DK, Papile LA, Poole WKI: Sepsis in very low birth weight neonates; the experience of the NICHD Neonatal Research Network. Pediatrics 2002, 110:285-291.

2. Lottspeich F, Kellermann J, Keidel EM: Molecular biology tools: proteomics techniques in biomarker discovery. Scand J Clin Lab Invest Suppl 2010, 242:19-22.

3. Ng PC, Ang IL, Chiu RWK, Li K, Lam S, Wong, RPO, Chui KM, Cheung HM, Ng EWY, Fok TF, Sung JJY, Lo YMD, Poon TCW: Host response biomarkers for diagnosis of late onset septicemia and necrotizing enterocolitis in preterm infants. J Clin Invest 2010, 120:2989-3000.

4. Poitou C, Viguerie N, Cancello R, De Matteis R, Cinti S, Stich V, Coussieu C, Gauthier E, Courtine M, Zucker JD, Barsh GS, Saris W, Bruneval P, Basdevant A, Langin D, Clément K: Serum amyloid A: production by human white adipocyte and regulation by obesity and nutrition. Diabetologia 2005, 48:519-528.

5. Yamada T, Miyake N, Itoh K, Igari J: Further characterization of serum amyloid $\mathrm{A} 4$ as a minor acute phase reactant and a possible nutritional marker. Clin Chem Lab Med 2001, 39:7-10.

6. Iriyama K, Nishiwaki H, Terashima H, Tonouchi H, Miki C, Suzuki H, Carpentier YA: Apolipoprotein C-II modifications associated with an infusion of artificial lipid particles. JPEN J Parenter Enteral Nutr 1988, 12:60-62.

7. Murch O, Collin M, Hinds, CJ, Thiemerman C: Lipoproteins in inflammation and sepsis. I. Basic Science. Intensive Care Med 2007, 33:13-24.

8. Samra JS, Summers KM, Frayn KN: Sepsis and fat metabolism. Br J Surg 1996, 83:1186-1196.

9. Liberatori A, Bini L, De Felice C, Magi B, Marzocchi B, Reggiaschi R, Pallini V, Bracci R: Acute phase proteins in perinatal plasma. Electrophoresis 1997, 18:520-526. 
10. Malle E, Sodin-Semri S, Kovacevic A: Serum amyloid A. An acute phase protein involved in tumor pathogenesis. Cell Mol Life Sci 2009, 66:9-26.

11. Arnon S, Litmanovitz I, Regev, R, Bauer S, Lis M, Shainkin-Kestenbaum R, Dolfin T: Serum amyloid A protein is a useful inflammatory marker during late onset sepsis in preterm infants. Biol Neonate 2005, 87:105-110.

12. Agranoff D, Fernandez-Reyes D, Papadopoulos MC, Rojas SA, Herbster M, Loosemore A, Tarelli E, Sheldon J, Schwenk A, Pollok R, Rayner CF, Krishna S:
Identification of diagnostic markers for tuberculosis by proteomic fingerprinting of serum. Lancet 2006, 368:1012-1021.

doi:10.1186/gm179

Cite this article as: Polin RA, Randis TM: Biomarkers for late-onset neonatal sepsis. Genome Medicine 2010, 2:58 